\title{
Soins Prénatals et Accouchement Assisté en Guinée
}

\author{
Balde Mamadou Saliou, \\ Collaborative PhD Program (CPP)-African Economic Research Consortium \\ (AERC)-Kenya. Université Felix Houphouët Boigny-Abidjan \\ Ministère de l'Enseignement Supérieur et de la Recherche Scientifique- \\ UGLC-Guinée. Direction Nationale des Etudes Economiques et de la \\ Prévision (DNEEP), Guinée
}

Doi:10.19044/esj.2020.v16n16p21 ～URL:http://dx.doi.org/10.19044/esj.2020.v16n16p21

\section{Résumé}

Des objectifs du millénaire pour le développement (OMD) aux objectifs de développement durable (ODD), la santé maternelle occupe une place importante. En Guinée, la situation sanitaire des femmes enceintes demeure une préoccupation majeure. En effet, le taux de mortalité maternelle se situait à 576 décès maternels pour 100000 naissances vivantes en 2017. Les principales causes de cette mortalité maternelle élevée est la nonutilisation adéquate des services de soins prénatals ou l'absence d'assistance médicale à l'accouchement. L'objectif de cette étude est d'identifier les déterminants de la demande de soins prénatals, et de voir l'effet de ces soins sur l'accouchement assisté. Pour ce faire, les données utilisées sont celles de l'enquête démographique et de santé (EDS V). Elle a été conduite par l'institut national de la statistique (INS) avec l'assistance technique de la société ICF international. Cette étude utilise un modèle négatif binomial pour identifier les déterminants de la demande de soins prénatals, et une régression logistique pour voir l'effet de ces soins sur l'accouchement assisté. Les résultats montrent que les déterminants de la demande de soins prénatals sont : le niveau de vie du ménage, l'instruction de la femme et de son conjoint, le milieu de résidence, l'exposition de la femme à la télévision et la souscription à une assurance. Aussi, ils confirment l'effet positif des soins prénatals sur le recours à l'accouchement assisté. L'étude recommande la sensibilisation sur les avantages de la consultation prénatale et l'accouchement assisté sur la réduction de la morbidité et la mortalité maternelle. Aussi, la gratuité des consultations prénatales et la réduction des frais d'accouchement afin d'augmenter leurs utilisations.

Mots clés : Soins prénatals, accouchement assisté, négatif binomial, Guinée 


\title{
Prenatal Healthcare and Assisted Delivery in Guinea
}

\author{
Balde Mamadou Saliou, \\ Collaborative PhD Program (CPP)-African Economic Research Consortium \\ (AERC)-Kenya. Université Felix Houphouët Boigny-Abidjan \\ Ministère de l'Enseignement Supérieur et de la Recherche Scientifique- \\ UGLC-Guinée. Direction Nationale des Etudes Economiques et de la \\ Prévision (DNEEP), Guinée
}

\begin{abstract}
From the millennium development goals (MDGs) to the sustainable development goals (SDGs), maternal health occupies an important place. In Guinea, the health situation, particularly that of pregnant women, remains a major concern. The maternal mortality rate stood at 576 maternal deaths per 100,000 live births in 2017. Most of the medical causes of maternal mortality are the lack of adequate use of prenatal healthcare services or the lack of medical assistance at delivery. This paper aims at identifying the driving factors of the demand for antenatal healthcare and medical assistance at delivery. The study looks at the effect of antenatal healthcare on assisted childbirth in Guinea. The study used data from the 2018 wave of the demographic and health survey (DHS V) in Guinea conducted by the national institute of statistics (INS) with technical support from macro international. The paper employs a negative binomial model for the prenatal healthcare and a logit regression to assess the relationship between prenatal healthcare and assisted childbirth. The findings of the study indicate that the standard of living, education, residential milieu, exposure to women's media and insurance coverage are the most important determinants of the demand for antenatal healthcare. The results also confirm the positive effect of prenatal healthcare on the use of assisted childbirth. The paper recommends raising women's awareness of the advantages and benefits of prenatal consultation and assisted childbirth. Also, antenatal consultations should be made free of charge and delivery fees should be reduced to increase their use.
\end{abstract}

Keywords: Prenatal healthcare, assisted delivery, negative binomial, Guinea

\section{Introduction}

Les questions relatives à la santé maternelle demeurent toujours une préoccupation dans les pays en développement (Bauserman et al., 2015). 
Selon l'organisation mondiale de la santé (OMS), environ 830 femmes meurent chaque jour de causes évitables liées à la grossesse et à l'accouchement. Aussi, 7000 nouveau-nés décèdent au cours du premier jour ou de la première semaine de leur vie. La quasi-totalité des décès maternels (99\%) et infantiles (98\%) surviennent dans les pays à faible revenu (OMS, 2019).

En République de Guinée, le taux de mortalité maternelle se situait à 576 pour 100000 naissances vivantes en 2017 (EDS, 2018). En effet, même si l'initiative de Bamako (IB) ${ }^{1}$ a permis d'élargir la couverture sanitaire nationale, de nombreux obstacles persistent encore empêchant les plus pauvres à l'accès aux soins (Doumbouya, 2008). La demande de soins dépasse largement l'offre, et les populations deviennent réticentes de payer pour accéder à des services de soins de mauvaise qualité. Ces comportements réduisent les fréquentations des hôpitaux et autres unités de soins, intensifiant ainsi les pratiques de santé informelles (Audibert et al., 2004). Avec l'avènement de la pandémie du Covid-19, les femmes seront encore beaucoup plus réticentes à l'idée d'aller effectuer des visites prénatales.

La plupart des décès maternels et néonataux peuvent être évités par la mise en place d'interventions telles que les soins prénatals et l'assistance qualifiée à l'accouchement (Lassi et al., 2010). Cependant, en Afrique subsaharienne, où les deux tiers des décès maternels mondiaux surviennent, c'est seulement $60 \%$ des naissances qui étaient assistées par un personnel de santé qualifié (ONU, 2019). Bien qu'en Guinée, des efforts soient faits pour promouvoir l'accouchement dans les établissements de santé, l'accouchement à domicile demeure encore assez fréquent avec $45 \%$ en 2018 (EDS, 2018). Par ailleurs, dans les régions en développement, $63 \%$ des femmes enceintes ont bénéficié de quatre consultations prénatales ou plus en 2017 ; allant de 51 $\%$ des femmes enceintes en Afrique à $88 \%$ en Amérique latine et dans les Caraïbes. Toutefois, $37 \%$ des femmes n'ont pas reçu de soins prénatals adéquats ou n'en ont pas reçu du tout (English et al., 2017). En République de Guinée, alors que la nouvelle recommandation de l'organisation mondiale de la santé (OMS) passe de 4 à 8 consultations prénatales, le pourcentage des femmes ayant effectué quatre consultations prénatales a connu une baisse ; passant de $57 \%$ en 2012 à $35 \%$ en 2018. En sus, moins d'une femme sur trois a effectué sa première visite prénatale à un stade précoce de la grossesse, soit moins de 4 mois $(29 \%)$. Ce taux varie de $38 \%$ en milieu urbain à $24 \%$ en milieu rural (EDS Guinée, 2018).

\footnotetext{
${ }^{1}$ La Guinée a adhéré à l'initiative de Bamako (IB) en 1987. Cette initiative vise à collecter auprès des usagers des services sanitaires, des ressources additionnelles pour financer l'accès aux soins de santé primaire, à améliorer l'efficience des structures sanitaires et la qualité des prestations. En contrepartie de cette participation financière, la gestion de ces unités de soins est déléguée aux différentes communautés.
} 
L'intérêt de cette étude réside au fait que, peu d'études se sont intéressées aux soins de santé maternelles en Guinée. Les quelques études réalisées dans ce domaine sont, pour la plupart, de nature descriptive (Doumbouya, 2008). Il est a noté qu'il n'existe aucune étude en Guinée qui s'est attelée à évaluer l'effet des soins prénatals sur le recours à l'accouchement assisté par un personnel de santé qualifié. Cette étude a pour objectif de combler cette insuffisance, ainsi d'identifier les déterminants de la demande de soins prénatals, et voir l'effet de ces soins sur l'accouchement assisté en Guinée.

\section{Revue de la littérature}

Les soins maternels regroupent un ensemble d'interventions dont le but est de prévenir ou d'identifier et de traiter les conditions pouvant compromettre la santé de la mère ou du fœetus/nouveau-né (OMS, 2003). Plusieurs études se sont intéressées aux déterminants de la demande de soins prénatals dans les pays en développement (Koné, 2019 ; Rwenge, 2014 ; Tiehi, 2013 ; Nanfosso \& kasiwa, 2013 ; Sirpe, 2011 ; Adjiwanou \& LeGrand, 2013) et de la relation entre soins prénatals et l'assistance médicale à l'accouchement (Ousseini, 2016 ; Ngom, 2016 ; Adjiwanou, 2011).

En effet, selon Ousseini (2016), le recours aux soins prénatals, la qualité des soins, le niveau de vie, l'instruction et la proportion de femmes avec un niveau secondaire impactent positivement le recours à l'accouchement assisté d'une part ; alors que, la taille du ménage, sa situation géographique, la proportion d'enfants de bas âge ont un effet négatif sur le recours à l'accouchement assisté au Niger d'autre part. De même, des études similaires menées au Burkina Faso (De Allegri et al., 2011) et dans une vingtaine de pays d'Afrique au sud du Sahara (Nikiéma et al., 2009) concluent que le nombre de visites reçues pendant la grossesse a un effet positif sur l'accouchement médicalisé. La consultation prénatale aurait donc la vertu d'éduquer les femmes sur leur besoin et de les familiariser avec les systèmes de santé moderne (Ahmed et al., 2010).

En Guinée, Diallo et al. (1999) ont analysé les problèmes médicaux et socioculturels de l'inadéquation entre les taux de consultations prénatales et l'accouchements assistés. Ils arrivent à la conclusion que, 67,30\% des femmes accouchent encore à domicile sans assistance en raison de l'éloignement des structures de santé, la pauvreté, l'analphabétisme, la mauvaise réception dans les établissements de santé, les coutumes et traditions. Dans le même ordre d'idées, selon Doumbouya (2008), malgré une nette amélioration de l'offre de soins de santé suite à l'application de l'initiative de Bamako (1987), de nombreux obstacles persistent en matière d'accessibilité des services de santé en Guinée. Parmi ces obstacles, il cite les carences institutionnelles, la qualité médiocre des services, le clientélisme dans les centres de santé, etc. Pour 
l'auteur, un engagement soutenu de l'État est suggéré pour consolider les infrastructures hospitalières et favoriser le bon fonctionnement du système de santé national.

S'agissant des déterminants de la demande de soins prénatals, Kochou \& Rwenge (2014) trouvent que l'ethnie, le degré de modernité, la distance et le niveau de vie du ménage expliquent dans une proportion de $60 \%$ les comportements de recours aux soins prénatals en Côte d'Ivoire. Cependant, plusieurs barrières socioculturelles empêchant le recours aux soins prénatals ont été identifiées en Côte d'Ivoire par Bouchon (2012). Il cite la perception de la grossesse comme n'étant pas une maladie, la méconnaissance des mesures préventives des pathologies liées à la grossesse et de leur symptomatologie, l'idée selon laquelle une femme enceinte doit vivre sa grossesse en toute discrétion, la multitude des interdits pour la femme enceinte et la perception de certaines pathologies comme magico-religieuses. Il conclut à l'existence d'un grand écart entre normes médicales et attitudes ou pratiques socioculturelles en République de Côte d'Ivoire tout comme partout ailleurs en Afrique subsaharienne.

De même, selon Sirpe (2011), la demande de soins prénatals au Burkina est essentiellement déterminée par le niveau de vie du ménage et le coût des soins. Pour lui, les soins prénataux peuvent être promue à travers l'amélioration des infrastructures de transport et de communication, comme le montre l'expérience Ougandaise en milieu rural (Musoke, 2002). De plus, l'auteur affirme que l'attitude des femmes face aux soins prénataux semble être influencée par leur niveau de scolarisation. Par contre, la religion a peu d'effet sur le recours aux soins prénataux. Pour Cissé (2002), en Afrique subsaharienne, l'accès aux soins de santé dépend dans la plupart des cas, non seulement de l'état de santé tel que perçu par l'individu, mais également des facteurs économiques tels que le revenu, le prix des services et des biens, la distance et les coûts de transport.

\section{Méthodologie de l'étude}

\subsection{Données et variables de l'étude}

Cette étude utilise les données de l'enquête démographique et de santé réalisée en Guinée en 2018. Elle a été conduite par l'Institut National des Statistiques (INS) avec l'assistance technique de la société ICF International. Des informations ont été collectées auprès de 7912 ménages composés de 10874 femmes âgées de 15 à 49 ans et 4117 hommes. Le territoire national a été découpé en 8 domaines d'étude correspondant aux 7 régions administratives et la capitale Conakry. Dans chaque domaine d'étude (sauf la ville de Conakry), deux strates ont été créées : la strate du milieu urbain et celle du milieu rural. Au total, 15 strates d'échantillonnage ont été créées. L'échantillon de premier degré est tiré indépendamment dans chaque strate, et 
celui de second degré est aussi tiré indépendamment dans chaque unité primaire tirée au premier degré.

La plupart des études portant sur le recours aux soins de santé maternelle se sont basées soit sur les déterminants liés à la demande ou ceux liés à l'offre. Cette étude s'inspire du cadre conceptuel développé par Beninguisse (2001) qui propose « une approche intégrée offre-demande ». Les facteurs liés à la demande regroupent entre autres, les facteurs économiques (le revenu), les facteurs culturels (ethnie, religion), les facteurs socio démographiques (état matrimonial, âge et parité atteinte) et les facteurs liés au rapport genre. Les facteurs liés à l'offre de soins sont entre autres, la qualité des soins, l'accessibilité économique et géographique des services de soins de santé.

Dans cette étude, deux variables dépendantes seront analysées. Il s'agit du nombre de consultations prénatales (variable comptable) et l'accouchement assisté qui est dichotomique pour chaque type d'assistance (Docteur, Infirmier ou les Agents Techniques de la Santé). Les variables sociodémographiques sont : l'âge de la femme, la parité (Fotso, Ezeh \& Oronje, 2008), le niveau d'éducation (Raghupathy, 1996), l'occupation de la femme, son statut matrimonial, sa religion et le sexe du chef de ménage. Le niveau d'éducation du mari, l'exposition de la femme à la télévision, son milieu de résidence et sa souscription à une mutuelle d'assurance ont été aussi prises en compte dans l'étude.

\subsection{Spécification des modèles économétriques}

Le premier objectif de cette étude est d'identifier les déterminants de la demande de soins prénatals en Guinée. Pour ce faire, elle a considéré le nombre de visites prénatales effectuées par la femme enceinte auprès du personnel soignant comme variable endogène (Conway \& Deb, 2005 ; Winkelmann, 2006). Le modèle de régression de Poisson (Hilbe, 2007) est souvent retenu pour expliquer une variable quantitative à valeurs entières. Ainsi, la probabilité que le nombre de visites prénatales prennent les valeurs $\left(y_{i}=0,1,2 \ldots\right)$ est :

$P\left(Y_{i}=y_{i}\right)=f\left(y_{i} \mid x_{i}\right)=\frac{e^{-\lambda_{i}} \lambda_{i}^{y_{i}}}{y_{i} !}$

Où $\lambda$ qui est positif est le paramètre de la distribution de Poisson, tel que $: E\left(y_{i}\right)=\operatorname{var}\left(y_{i}\right)=\lambda$. Ce paramètre $\lambda_{i}$ dépend du vecteur de co-variables $X_{i}$ par une équation $\log$ linéaire notée comme suit $: \log \lambda_{i}=x_{i} \beta$. Le choix de la spécification log-linéaire s'explique essentiellement par la nécessité d'avoir des paramètres $\lambda_{i}$ positifs. Cependant, du fait d'une abondance de valeurs nulles et/ou de la présence de quelques valeurs extrêmes, la variance est souvent supérieure à la moyenne. Dans ce cas, on parle d'une sur-dispersion 
(Cox, 1983 ; Hinde \& Demétrio, 1998) de la variable endogène. Pour pallier à cela, cette étude utilise un modèle de comptage alternatif basé sur la loi binomiale négative, qui permet de tenir en compte cette sur-dispersion par l'introduction d'un paramètre supplémentaire $\alpha$. Ce paramètre permet de capter l'hétérogénéité inobservée de la variable endogène (qui peut impliquer la sur-dispersion inobservée). Dans cette modélisation, $y_{i}$ suit toujours une loi de Poisson de paramètre :

$\mu_{i}=e^{\left(x_{i} \beta+\varepsilon_{i}\right)}=\lambda_{i} e^{\varepsilon_{i}}$

Avec $e^{\varepsilon_{i}}=u_{i}$. Ainsi, la probabilité que le nombre de visites prénatales prennent les valeurs $\left(y_{i}=0,1,2 \ldots \ldots\right)$ est :

$P\left(Y_{i}=y_{i}\right)=f\left(y_{i} \mid x_{i}\right)=\frac{e^{-\lambda_{i} u_{i}}\left(\lambda_{i} u_{i}\right)^{y_{i}}}{y_{i} !}$

Mais la densité de $y_{i}$ sachant $x_{i}$ est obtenue en prenant l'espérance de l'expression ci-dessus par rapport à la densité de $u_{i}$ :

$$
\begin{aligned}
P\left(Y_{i}=y_{i}\right)=f\left(y_{i} \mid x_{i}\right) & =\int_{0}^{\infty} \operatorname{Prob}\left(Y_{i}=y_{i} / x_{i}, u_{i}\right) g\left(u_{i}\right) d u_{i} \\
& =\int_{0}^{\infty} \frac{e^{-\lambda_{i} u_{i}}\left(\lambda_{i} u_{i}\right)^{y_{i}}}{y_{i} !} g\left(u_{i}\right) d u_{i} \ldots \ldots \ldots
\end{aligned}
$$

Cet article suppose que $u_{i}$ suit une loi gamma $\gamma(\delta, \delta), \delta>0$ de densité $g\left(u_{i}\right)$, d'espérance $E\left(u_{i}\right)=1$ et de variance $\operatorname{Var}\left(u_{i}\right)=1 / \delta$. Avec cette normalisation,

$$
\begin{aligned}
g\left(u_{i}\right)=\frac{\delta^{\delta}}{\Gamma(\delta)} e^{-\delta u_{i}} u_{i}^{\delta-1} & \\
P\left(Y_{i}=y_{i}\right)=f\left(y_{i} \mid x_{i}\right) & =\frac{e^{-\lambda_{i} u_{i}}\left(\lambda_{i} u_{i}\right)^{y_{i}}}{y_{i} !} \frac{\delta^{\delta}}{\Gamma(\delta)} e^{-\delta u_{i}} u_{i}^{\delta-1} d u_{i} \\
& =\frac{\delta^{\delta} \lambda_{i}^{y}}{\Gamma(y+1) \Gamma(\delta)} \int_{0}^{\infty} e^{-\left(\lambda_{i}+\delta\right) u_{i}} u_{i}^{\delta+y-1} d u_{i} \\
& =\frac{\delta^{\delta} \lambda_{i}{ }^{y} \Gamma(\delta+y)}{\Gamma(y+1) \Gamma(\delta)\left(\lambda_{i}+\delta\right)^{\delta+y}} \\
& =\frac{\Gamma(\delta+y)}{\Gamma(y+1) \Gamma(\delta)}\left[\frac{\lambda_{i}}{\lambda_{i}+\delta}\right]^{y}\left[\frac{\delta}{\lambda_{i}+\delta}\right]^{\delta} \cdots \cdots \cdots
\end{aligned}
$$

Le modèle négatif binomial peut être estimé par le maximum de vraisemblance. La spécification de Poisson est testée par l'hypothèse nulle : $H_{0}: \delta=0$. Le test du rapport de vraisemblance est utilisé à cet effet (Greene, 2000). Le second objectif de l'étude examine la relation entre les soins prénatals et l'accouchement assisté par un personnel de santé qualifié (docteur, infirmier et ATS). De ce fait, à partir des résultats du test IIA, une régression logistique sera utilisée. Le logit est spécifié comme suit : 
Soit $y_{i}^{*}=x_{i}^{\prime} \beta+\varepsilon$

$$
\begin{aligned}
\operatorname{Pr}\left(y_{i}=1 / x_{i}\right)= & \operatorname{Pr}\left(y_{i}^{*}>0 / x_{i}\right) \ldots \ldots \ldots \ldots \\
& =\operatorname{Pr}\left(x_{i}^{\prime} \beta+\varepsilon_{i}>0 / x_{i}\right) \\
& =\phi\left(x_{i}^{\prime} \beta\right) \\
\operatorname{Pr}\left(y_{i}=0 / x_{i}\right)= & 1-\phi\left(x_{i}^{\prime} \beta\right) \ldots \ldots \ldots \ldots \ldots
\end{aligned}
$$

\section{Résultats et discussions}

\subsection{Déterminants de la demande de soins prénatals}

Les estimations des modèles de Poisson et négatif binomial (Tableau 1) confirment la supériorité du modèle négatif binomial. Le test du ratio de vraisemblance $($ Prob $>=$ chibar $2=0.000$ ) conduit au choix du modèle négatif binomial. Il ressort des estimations que le niveau d'éducation de la femme et de son mari, la religion musulmane, le niveau de revenu du ménage, le milieu de résidence, le sexe du chef de ménage, l'assurance, l'opportunité de la grossesse, l'exposition de la femme à la télévision, l'âge de la femme à la naissance et son statut marital expliquent la demande de soins prénatals en République de Guinée.

Á cet effet, les effets marginaux du modèle négatif binomial (Tableau 1) montrent qu'une variation d'une année supplémentaire d'instruction primaire, secondaire et supérieure des femmes augmente, respectivement, le nombre de consultations prénatales de $0,26,0,52$ et 0,54 points, toutes choses égales par ailleurs, comparées à celles ne disposant aucun niveau. Les mêmes résultats sont obtenus, lorsqu'on contrôle par l'instruction de son conjoint, avec une augmentation de 0,39 points suite à une année supplémentaire d'éducation. Ces résultats sont similaires à ceux obtenus par d'autres auteurs (Koné, 2019 ; Rwenge, 2014 ; Nanfosso et kasiwa, 2013 ; Sirpe, 2011 ; Nadjihoroum, 2007 ; Jaffré \& Prual, 1993 et 1994, Beninguissé, 2001 ; Raghupathy, 1996 ; Adjiwanou \& LeGrand, 2013 ; Tiehi, 2013). L'instruction de la femme augmente donc son pouvoir de décision et ces contacts avec le monde extérieur. De même, l'exposition de la femme à la télévision a un effet positif et significatif sur la demande de soins prénatals avec un effet marginal de 0,38. Le même résultat a été obtenu au Congo par Nanfosso \& Kasiwa (2013). La religion musulmane a également un effet positif et significatif sur la demande de soins prénatals. La pratique de la religion musulmane par les femmes est associée à une augmentation du nombre des visites prénatales de 0,42 points. Ces résultats sont similaires à ceux obtenus par Koné (2019) pour la Cote d'Ivoire, Nanfosso \& kasiwa (2013) pour le Congo. D'après plusieurs auteurs faisant référence au Coran, le Prophète Mahomet (PSL) a dit qu'il faut prendre soins de la santé de soi et de la famille et prendre des mesures de précaution pour prévenir les maladies (Al Munâjid, 2019 ; cité par Koné, 2019). 
Il en va de même pour le milieu de résidence. Le nombre de consultions prénatales augmente de 0,68 points pour les femmes vivant en milieu urbain comparées à celles vivant en milieu rural. En milieu urbain, les femmes ont plus accès aux structures sanitaires. Ces mêmes résultats sont obtenus par (Koné, 2019 ; Beninguissé, 2001, Akoto et al., 2002 ; Mazalale et al., 2015 ; Dickson, Adde, \& Amu, 2016). Aussi, au fur et à mesure que le statut économique du ménage s'améliore, passant des pauvres aux riches, le nombre de visites prénatales augmente de 0,17 et 0,19 points respectivement. Ces résultats sont comparables à ceux obtenus par (Mbugua \& MacQuarrie, 2018 ; Beninguissé et al., 2003 ; Rwenge, 2007 ; Sawadogo et al., 2012 ; Akoto et al., 2002). Le sexe du chef de ménage exerce une influence significative sur les soins prénatals. Ainsi, les effets marginaux du modèle négatif binomial indiquent que les femmes vivant dans des ménages dirigés par elles même sont plus enclines à demander des soins prénatals (avec un effet marginal de 0,24). Ce résultat est similaire à celui de Yana (1993). Aussi, le fait d'être en couple, de souscrire à une assurance augmente le nombre de visites prénatales de 0,34 et 0,42 points respectivement. Ce résultat est similaire à celui obtenu par Nanfosso \& kasiwa (2013) pour le Congo. De même, une synthèse de la littérature théorique et empirique sur l'effet des dépenses sur l'utilisation des soins de santé effectuée par Youness, Houda, \& Hicham, (2018) conclut à l'existence d'une relation positive entre l'accès à l'assurance maladie et la consommation de soins de santé. Également, le fait que la grossesse ait été voulue au moment de sa survenue est associé à une augmentation du nombre de visites prénatales de 0,52 points au seuil de $1 \%$. Par contre, l'âge à la naissance est associé à une réduction du nombre de visites prénatales de 0,01 points. Cela s'explique par le fait que, les femmes âgées utilisent moins les soins prénatals que celles d'âge inférieur (Ochako, 2003).

Tableau 1 : Résultats de l'estimation des modèles négatif binomial et Poisson

\begin{tabular}{lll}
\hline Variables & Négatif binomial & \multicolumn{1}{c}{ Poisson } \\
\cline { 2 - 3 } Primaire & Effets marginaux & Effets marginaux \\
& $0,2691826^{* * *}$ & $0,2693131^{* * *}$ \\
Secondaire & $(0,09178)$ & $(0,09129)$ \\
& $0,5221717 * * *$ & $0,5114011^{* * *}$ \\
Supérieur & $(0,10239)$ & $(0,10191)$ \\
& $0,5458082^{* *}$ & $0,5295162 * *$ \\
Musulmane & $(0,23364)$ & $(0,2287)$ \\
Autres religions & $0,4284683^{* *}$ & $0,4083801 * *$ \\
& $(0,17173)$ & $(0,17216)$ \\
Très pauvre & 0,2521243 & 0,2457572 \\
Pauvre & $(0,2364)$ & $(0,23359)$ \\
Revenu moyen & 0,1458213 & 0,1360277 \\
& $(0,09655)$ & $(0,0951)$ \\
& $0,1713747 *$ & $0,1692773 *$ \\
& $(0,09854)$ & $(0,09713)$ \\
& 0,1500025 & 0,1490639
\end{tabular}




\begin{tabular}{|c|c|c|}
\hline & $(0,0999)$ & $(0,09845)$ \\
\hline \multirow[t]{2}{*}{ Riche } & $0,1759962 *$ & $0,1676726^{*}$ \\
\hline & $(0,09737)$ & $(0,09539)$ \\
\hline \multirow[t]{2}{*}{ Soussou } & 0,1677416 & 0,1759603 \\
\hline & $(0,19881)$ & $(0,199)$ \\
\hline \multirow[t]{2}{*}{ Peulh } & $-0,1897367$ & $-0,1530457$ \\
\hline & $(0,18539)$ & $(0,18603)$ \\
\hline \multirow[t]{2}{*}{ Malinké } & $-0,1904096$ & $-0,159832$ \\
\hline & $(0,18523)$ & $(0,18611)$ \\
\hline \multirow[t]{2}{*}{ Milieu de résidence } & $0,6822399 * * *$ & $0,6725987 * * *$ \\
\hline & $(0,08022)$ & $(0,08045)$ \\
\hline \multirow[t]{2}{*}{ Sexe chef du ménage } & $0,2452891 * *$ & $0,2306726 * *$ \\
\hline & $(0,09083)$ & $(0,08935)$ \\
\hline \multirow[t]{2}{*}{ Assurance } & $0,4227468 * *$ & $0,420987 * *$ \\
\hline & $(0,20223)$ & $(0,2003)$ \\
\hline \multirow[t]{2}{*}{ Opportunité de la grossesse } & $0,5219321 * * *$ & $0,5148579 * * *$ \\
\hline & $(0,08685)$ & $(0,08709)$ \\
\hline \multirow[t]{2}{*}{ Exposition à la télévision } & $0,3839059 * * *$ & $0,3839487 * * *$ \\
\hline & $(0,07596)$ & $(0,07589)$ \\
\hline \multirow[t]{2}{*}{ Education du conjoint } & $0,3976751 * * *$ & $0,3945775^{* * *}$ \\
\hline & $(0,06895)$ & $(0,06904)$ \\
\hline \multirow[t]{2}{*}{ Occupation de la femme } & $-0,0178118$ & $-0,0132955$ \\
\hline & $(0,06192)$ & $(0,00706)$ \\
\hline \multirow[t]{2}{*}{ Age à la naissance } & $-0,0138618 *$ & $-0,0186199 *$ \\
\hline & $(0,00712)$ & $(0,06118)$ \\
\hline \multirow[t]{2}{*}{ Statut marital } & $0,3468738 * *$ & $0,3384816^{* * *}$ \\
\hline & $(0,16597)$ & $(0,16404)$ \\
\hline \multicolumn{3}{|c|}{$\begin{array}{c}\text { Notes : écart type entre parenthèses ; } * * * p<0.01, * * p<0.05, * p<0.1 \text {. Test du rapport des } \\
\text { vraisemblances de alpha }(\alpha)=0: \text { chibar } 2(01)=113.74 \text { Prob }>=\text { chibar } 2=\mathbf{0 . 0 0 0}\end{array}$} \\
\hline
\end{tabular}
Source : auteur, à partir de la base de données EDS Guinée, 2018

\subsection{Effet des soins prénatals sur l'accouchement assisté}

La régression logistique confirme l'effet positif des soins prénatals sur l'accouchement assisté. En effet, les femmes ayant effectué au moins une consultation prénatale au cours de la grossesse ont 1,18 fois plus de chance d'être assistée par un Docteur, 1,35 fois plus de chance de l'être par un Infirmier et 1,15 fois par un ATS. Ces résultats sont conformes à ceux obtenus par (Ousseini, 2016 ; Ngom, 2016 ; Adjiwanou, 2013 ; Taye \& Belachew, 2016 ; Dickson, Adde \& Amu, 2016 ; Owili et al., 2016). Les résultats montrent également que l'instruction de la femme et de son mari a un effet positif sur l'accouchement assisté. Les femmes avec un niveau primaire, secondaire et supérieur ont 1,43, 1,51 et 2,46 fois, respectivement, plus de chance d'être assisté par un Docteur au moment de l'accouchement. De même, les femmes avec un niveau d'éducation primaire ont 1,44 fois plus de chance d'être assisté par Infirmier, et celles ayant un niveau secondaire ont 1,42 fois plus de chance de l'être par un Infirmier. Ces résultats sont conformes à la littérature précédente (Ousseini, 2016 ; Adjiwanou, 2011 ; Alderman et al., 
2003). Selon Harouna (1996, cité par Koné, 2019) : « les mères instruites sont matériellement et socialement plus proches des services de soins de santé modernes que leurs congénères non instruites ».

L'assistance à l'accouchement augmente aussi avec le degré de citadinité. Ainsi, les femmes vivant en milieu urbain ont 1,49 fois plus de chance d'être assisté par un Docteur et 1,29 fois plus de chance d'être assisté par un Infirmier que celles du milieu rural. En effet, les femmes rurales trouvent des avantages à accoucher à domicile avec l'aide des personnels de santé non formés (accoucheuses traditionnelles, les guérisseurs, amis et parents). Les raisons sont : les coûts des soins qui sont plus abordables, les personnels de santé à portée de main (accessibilité économique et géographique). De ce fait, l'accouchement se passe dans un cadre plus convivial et intime, on respecte les coutumes de protection et de purification de la mère et du bébé (Béninguissé, 2001 ; Adjamagbo et al., 1999). Les mêmes résultats sont obtenus au Niger par Ousseini (2016). Par contre, les femmes qui vivent en milieu urbain ont 0,27 fois moins de chance d'être assisté par un agent technique de santé (ATS). Les femmes avec un mari disposant au moins d'une éducation primaire ont 1,63 plus de chance d'être assistées par un Docteur et 1,38 fois plus de chance de l'être par un ATS.

De même, les femmes qui ont souscrit à une assurance ont 2,09 fois plus de chance d'être assisté par un Docteur que celles n'ayant pas souscrit. Les ménages dirigés par un homme ont 0,70 fois moins de chance d'être assisté par un Infirmier que ceux dirigé par une femme. Les estimations montrent également que les femmes d'ethnie Peulh ont 0,53 fois moins de chance de voir un Infirmier que les femmes des autres ethnies. Dans le même sens, les femmes qui suivent la télévision ont 1,34 fois plus de chance d'être assisté par un Docteur, 1,33 fois plus de chance d'être assisté par un Infirmier et 1,46 fois plus de chance de l'être par un ATS. Les femmes ayant conçue une grossesse non désirée ont 0,70 fois moins de chance d'être assistée à l'accouchement. Les femmes âgées ont 0,97 fois moins de chance d'être assisté à l'accouchement que les jeunes femmes.

Tableau 2 : Coefficients et Odds ratio de l'effet des soins prénatals sur l'accouchement assisté.

\begin{tabular}{|c|c|c|c|c|c|c|}
\hline \multirow[t]{2}{*}{ Variables } & \multicolumn{2}{|l|}{ Docteur } & \multicolumn{2}{|l|}{ Infirmier } & \multicolumn{2}{|l|}{ ATS } \\
\hline & Coefficients & $\mathbf{O R}$ & Coefficients & $\mathbf{O R}$ & $\begin{array}{l}\text { Coeffici } \\
\text { ents }\end{array}$ & $\mathbf{O R}$ \\
\hline $\begin{array}{l}\text { Nombre de visites } \\
\text { prénatales }\end{array}$ & $\begin{array}{l}0,16636 * * * \\
(0,030831)\end{array}$ & $1,18 * * *$ & $\begin{array}{l}0,301177 * * * \\
(0,0220477)\end{array}$ & $1,35 * * *$ & $\begin{array}{r}0,14 * * * \\
(0,0273)\end{array}$ & $1,15 * * *$ \\
\hline \multicolumn{7}{|c|}{ Niveau d'éducation (référence : aucune éducation) } \\
\hline Primaire & $\begin{array}{l}0,3625782 * \\
(0,188040)\end{array}$ & $1,43 *$ & $\begin{array}{l}0,365122 * * * \\
(0,1344901)\end{array}$ & $1,44 * * *$ & $\begin{array}{r}0,13539 \\
(0,1966)\end{array}$ & 1,14 \\
\hline Secondaire & $\begin{array}{c}0,416746^{* *} \\
(0,1869087)\end{array}$ & $1,51 * *$ & $\begin{array}{l}0,3537611 * * \\
(0,1590261)\end{array}$ & $1,42 * *$ & $\begin{array}{l}-0,3201 \\
(0,231)\end{array}$ & 0,72 \\
\hline
\end{tabular}




\begin{tabular}{|c|c|c|c|c|c|c|}
\hline Supérieur & $\begin{array}{l}0,903428 * * * \\
(0,3161047)\end{array}$ & $2,46 * * *$ & $\begin{array}{r}-0,1372041 \\
(0,3674613)\end{array}$ & 0,87 & $\begin{array}{l}0,13125 \\
(0,476)\end{array}$ & 1,14 \\
\hline \multicolumn{7}{|c|}{ Ethnies (réf : ethnies forestière) } \\
\hline Soussou & $\begin{array}{l}-0,1616501 \\
(0,4184298)\end{array}$ & 0,85 & $\begin{array}{r}-0,1195274 \\
(0,2928578)\end{array}$ & 0,88 & $\begin{array}{l}-0,6882 * \\
(0,4017)\end{array}$ & $0,50 *$ \\
\hline Peulh & $\begin{array}{r}0,1529419 \\
(0,4020584)\end{array}$ & 1,16 & $\begin{array}{r}-0,61874 * * \\
(0,2840417)\end{array}$ & $0,53 * *$ & $\begin{array}{l}-0,5260 \\
(0,3833)\end{array}$ & 0,59 \\
\hline Malinké & $\begin{array}{l}-0,5849746 \\
(0,4203891)\end{array}$ & 0,55 & $\begin{array}{c}-0,1182725 \\
(0,2890206)\end{array}$ & 0,88 & $\begin{array}{l}-0,0533 \\
(0,3833)\end{array}$ & 0,94 \\
\hline \multicolumn{7}{|c|}{ Religion (réf : Chrétienne) } \\
\hline Musulmane & $\begin{array}{c}0,3910912 \\
(0,4553838)\end{array}$ & 1,47 & $\begin{array}{r}-0,3996103 \\
(0,2987991)\end{array}$ & 0,67 & $\begin{array}{c}-0,5606 \\
(0,3891)\end{array}$ & 0,57 \\
\hline Autres religions & $\begin{array}{l}-0,4211891 \\
(1,035909)\end{array}$ & 0,65 & $\begin{array}{r}-0,674264 * \\
(0,3816907)\end{array}$ & $0,50 *$ & $\begin{array}{c}0,4955 \\
(0,3755)\end{array}$ & 1,64 \\
\hline \multicolumn{7}{|c|}{ Niveau de richesse (réf : très riche) } \\
\hline Très pauvre & $\begin{array}{c}-0,199001 \\
(0,1951102)\end{array}$ & 0,81 & $\begin{array}{l}-0,1002826 \\
(0,1217763)\end{array}$ & 0,90 & $\begin{array}{c}0,1585 \\
(0,1820)\end{array}$ & 1,17 \\
\hline Pauvre & $\begin{array}{c}-0,0436893 \\
(0,1968789)\end{array}$ & 0,95 & $\begin{array}{c}0,1305693 \\
(0,122529)\end{array}$ & 1,13 & $\begin{array}{r}0,0456 \\
(0,1858)\end{array}$ & 1,04 \\
\hline Revenu moyen & $\begin{array}{l}0,0820355 \\
(0,1895855)\end{array}$ & 1,08 & $\begin{array}{r}-0,0633204 \\
(0,1286834)\end{array}$ & 0,93 & $\begin{array}{r}-0,2706 \\
(0,1960)\end{array}$ & 0,76 \\
\hline Riche & $\begin{array}{r}0,0522602 \\
(0,1925406)\end{array}$ & 1,05 & $\begin{array}{r}0,1719156 \\
(0,1264711)\end{array}$ & 1,18 & $\begin{array}{r}-0,1245 \\
(0,1942)\end{array}$ & 0,88 \\
\hline $\begin{array}{l}\text { Sexe chef de } \\
\text { ménage }\end{array}$ & $\begin{array}{c}0,0238925 \\
(0,1857968)\end{array}$ & 1,02 & $\begin{array}{l}-0,3533^{* * * *} \\
(0,130488)\end{array}$ & $0,70 * * *$ & $\begin{array}{r}-0,0850 \\
(0,1893)\end{array}$ & 0,91 \\
\hline $\begin{array}{l}\text { Occupation de la } \\
\text { femme }\end{array}$ & $\begin{array}{l}-0,3433727 * * * \\
(0,1321123)\end{array}$ & $0,70 * * *$ & $\begin{array}{r}0,0826659 \\
(0,0816024)\end{array}$ & 1,08 & $\begin{array}{l}0,07481 \\
(0,1247)\end{array}$ & 1,07 \\
\hline $\begin{array}{l}\text { Milieu de } \\
\text { résidence }\end{array}$ & $\begin{array}{l}0,6630391 * * * \\
(0,156838)\end{array}$ & $1,94 * * *$ & $\begin{array}{l}0,987521 * * * \\
(0,1073059)\end{array}$ & $1,29 * * *$ & $\begin{array}{l}-0,28 * * * \\
(0,1980)\end{array}$ & $0,27 * * *$ \\
\hline $\begin{array}{l}\text { Education du } \\
\text { conjoint }\end{array}$ & $\begin{array}{l}0,4930484 * * * \\
(0,1424797)\end{array}$ & $1,63 * * *$ & $\begin{array}{c}-0,0090668 \\
(0,0986894)\end{array}$ & 0,99 & $\begin{array}{l}0,32 * * * \\
(0,1425)\end{array}$ & $1,38 * *$ \\
\hline Age à la naissance & $\begin{array}{l}0,0197774 \\
(0,0151094)\end{array}$ & 1,01 & $\begin{array}{l}0,025881 \\
(0,0092989)\end{array}$ & 1,02 & $\begin{array}{l}-0,026^{*} \\
(0,0145)\end{array}$ & $0,97 *$ \\
\hline $\begin{array}{l}\text { Exposition } \\
\text { télévision }\end{array}$ & $\begin{array}{l}0,2897542^{*} \\
(0,1702294)\end{array}$ & $1,34 *$ & $\begin{array}{l}0,290308 * * * \\
(0,0947812)\end{array}$ & $1,33 * * *$ & $\begin{array}{l}0,38 * * * \\
(0,1467)\end{array}$ & $1,46^{* * *}$ \\
\hline $\begin{array}{l}\text { Opportunité de } \\
\text { grossesse }\end{array}$ & $\begin{array}{c}-0,0436778 \\
(0,1922786)\end{array}$ & 0,95 & $\begin{array}{r}0,1171827 \\
(0,1197653)\end{array}$ & 1,12 & $\begin{array}{l}-0,1547 \\
(0,167)\end{array}$ & 0,85 \\
\hline Gémellité & $\begin{array}{l}-0,4086758 \\
(0,3183893)\end{array}$ & 0,66 & $\begin{array}{c}-0,5308 * * * \\
(0,2305516)\end{array}$ & $0,58 * *$ & $\begin{array}{r}-0,0979 \\
(0,3509)\end{array}$ & 0,90 \\
\hline Statut marital & $\begin{array}{c}-0,1561498 \\
(0,4257894)\end{array}$ & 0,85 & $\begin{array}{r}-0,0936549 \\
(0,3083452)\end{array}$ & 0,91 & $\begin{array}{l}0,1825 \\
(0,3768)\end{array}$ & 1,20 \\
\hline Assurance & $\begin{array}{l}0,7414186^{*} \\
(0,4359525)\end{array}$ & $2,09 *$ & $\begin{array}{c}-0,5429188 \\
(0,5074714)\end{array}$ & 0,58 & $\begin{array}{r}-0,1831 \\
(0,6000)\end{array}$ & 0,83 \\
\hline
\end{tabular}

Notes : écart type entre parenthèses ; *** p<0.01, ** p<0.05, * p<0.1

Source : auteur, à partir de la base de données EDS Guinée, 2018

\section{Conclusions et recommandations}

L'objectif de cette étude était d'identifier les déterminants de la demande de soins prénatals, et de voir l'effet de ces soins sur l'accouchement 
assisté en Guinée. Les données de l'enquête démographique et de santé (EDS) ont été utilisées à cet effet. L'étude a aussi adopté un modèle négatif binomial pour les soins prénatals, et une régression logistique pour voir la relation entre ces soins et l'accouchement assisté par un personnel de santé qualifié. Il ressort des estimations que le niveau d'éducation de la femme et de son conjoint, le niveau de richesse du ménage, le milieu de résidence, le sexe du chef de ménage, l'exposition de la femme à la télévision, la souscription à une mutuelle d'assurance sont les déterminants les plus importants des soins prénatals. Ensuite, les résultats confirment l'effet positif des soins prénatals sur le recours à l'accouchement assisté en Guinée. Il en ressort aussi que les niveaux d'instruction de la femme et de son mari, les quintiles de bien-être et la proportion de femmes âgées d'au moins vingt ans avec un niveau d'éducation secondaire influencent positivement le recours à l'accouchement assisté.

Au regard de ces différents résultats, l'étude recommande la gratuité des services de consultations prénatales. Les pays dans lesquels les consultations prénatales ont été rendues gratuites ont vu augmenter le nombre d'accouchements assistés dans les formations sanitaires et une baisse du recours aux accoucheuses traditionnelles de manière considérable (Lagarde \& Palmer, 2006). En sus, pour faciliter l'accès aux soins prénatals en zones rurales, il faudrait promouvoir les activités mobiles de consultation prénatale à périodicité régulière. Les visites à domicile peuvent offrir une opportunité d'adaptation culturelle de la consultation prénatale et peuvent aussi faciliter l'engagement communautaire et l'interaction avec les accoucheuses traditionnelles. Un autre élément important est la sensibilisation médiatique sur les avantages et bénéfices de la consultation prénatale et l'accouchement assisté sur la réduction de la morbidité et la mortalité maternelle.

\section{References :}

1. Adjiwanou, V., \& LeGrand, T. (2013). Does antenatal care matter in the use of skilled birth attendance in rural Africa: a multi-country analysis. Social science \& medicine, 86, 26-34.

2. Akoto, E., \& Tambashe, B. (2002). Socioeconomic inequalities in infant and child mortality among urban and rural areas in sub-Saharan Africa. In First seminar of the IUSSP Committee on Emerging Health Threats, Max Planck Institute for Demographic Research, Rostock, 46.

3. Ali, K. J., \& Noman, A. (2013, November). Determinants of demand for health care in Bangladesh: an econometric analysis. In 3rd AsiaPacific Business Research Conference,2-4.

4. Aliou, D. J. F. D. (2017). Inférence statistique dans des modèles de comptage à inflation de zéro. Applications en économie de la 
santé (Doctoral dissertation, Rennes, Institut National des Sciences Appliquées), 22-25.

5. Andersen, R. (1968). A behavioral model of families' use of health services. A behavioral model of families' use of health services., (25).

6. Bauserman, M., Lokangaka, A., Thorsten, V., Tshefu, A., Goudar, S. S., Esamai, F., ... \& Manasyan, A. (2015). Risk factors for maternal death and trends in maternal mortality in low-and middle-income countries: a prospective longitudinal cohort analysis. Reproductive health, 12(2), S5.

7. Belachew, T., Taye, A., \& Belachew, T. (2016). Postnatal care service utilization and associated factors among mothers in Lemo Woreda, Ethiopia. J Women's Health Care, 5(10.4172), 2167-0420.

8. Belaid, L. (2015). Facteurs contextuels dans l'évaluation d'une politique de santé maternelle au Burkina Faso.

9. Beninguisse, G., Nikièma, B., Fournier, P., \& Haddad, S. (2005). L'accessibilité culturelle : une exigence de la qualité des services et soins obstétricaux en Afrique.

10. Cissé, B., Luchini, S., \& Moatti, J. P. (2004). Recouvrement des coûts et demande de soins dans les PED. Revue française d'économie, 18(4), 111-149.

11. Conway, K. S., \& Deb, P. (2005). Is prenatal care really ineffective? Or, is the 'devil'in the distribution? Journal of Health Economics, 24(3), 489-513.

12. Cox, D. R. (1983). Some remarks on overdispersion. Biometrika, 70(1), 269-274.

13. Darmstadt, G. L., Bhutta, Z. A., Cousens, S., Adam, T., Walker, N., De Bernis, L., \& Lancet Neonatal Survival Steering Team. (2005). Evidence-based, cost-effective interventions: how many newborn babies can we save? The Lancet, 365(9463), 977-988.

14. De Allegri, M., Ridde, V., Louis, V. R., Sarker, M., Tiendrebéogo, J., Yé, M., ... \& Jahn, A. (2011). Determinants of utilisation of maternal care services after the reduction of user fees: a case study from rural Burkina Faso. Health policy, 99(3), 210-218.

15. De-Banguirys, S. L., Dansou, J., \& Beninguisse, G. (2015). Analyse des facteurs de non recours en première Consultation Prénatale lors des premières grossesses des adolescentes/jeunes à Yaoundé. In 7th African Population Conference, Pretoria, South Africa, $18 p$.

16. Diallo, F. B., Diallo, A. B., Diallo, Y., Goma, O., Camara, Y., Cisse, M., \& Diallo, M. S. (1998). Mortalité maternelle et facteurs de risque liés au mode de vie. Médecine d'Afrique noire, 45(12), 723-727. 
17. Dickson, K. S., Adde, K. S., \& Amu, H. (2016). What influences where they give birth? Determinants of place of delivery among women in rural Ghana. International journal of reproductive medicine, 2016.

18. Dieng, M., Audibert, M., Le Hesran, J. Y., \& Dial, A. T. (2015). Déterminants de la demande de soins en milieu péri-urbain dans un contexte de subvention à Pikine, Sénégal.

19. Doumbouya, M. L. (2008). Accessibilité des services de santé en Afrique de l'Ouest : le cas de la Guinée.

20. Edgerley, L. P., El-Sayed, Y. Y., Druzin, M. L., Kiernan, M., \& Daniels, K. I. (2007). Use of a community mobile health van to increase early access to prenatal care. Maternal and Child Health Journal, 11(3), 235-239.

21. English, Philina A., Alexander M. Mills, Michael D. Cadman, Audrey E. Heagy, Greg J. Rand, David J. Green, and Joseph J. Nocera. "Eastern whip-poor-will migrations (data from English et al. 2017)." (2017).

22. Enquête Démographique et de Santé Guinée. (2018). Institut National des Statistiques (INS).

23. Fotso, J. C., Ezeh, A., \& Oronje, R. (2008). Provision and use of maternal health services among urban poor women in Kenya: what do we know and what can we do? Journal of Urban Health, 85(3), 428442.

24. Gourieroux, C. (1984). Econométrie des variables qualitatives. Paris: Economica.

25. Greene, R. L. (2000). The MMPI-2: An interpretive manual. Allyn \& Bacon.

26. Gupta, R., \& Talukdar, B. (2017). Frequency and Timing of Antenatal Care Visits and its Impact on Neonatal Mortality in EAG States of India. Journal of Neonatal Biology, 6(3), 263.

27. Hardin, J. W., Hardin, J. W., Hilbe, J. M., \& Hilbe, J. (2007). Generalized linear models and extensions. Stata press.

28. Jaffré, Y., \& Prual, A. (1993). « Le corps des sages-femmes », entre identités professionnelle et sociale. Sciences sociales et santé, 11(2), 63-80.

29. Jin, H. T., Anderson, A. C., Tan, W. G., West, E. E., Ha, S. J., Araki, K., ... \& Ahmed, R. (2010). Cooperation of Tim-3 and PD-1 in CD8 T-cell exhaustion during chronic viral infection. Proceedings of the National Academy of Sciences, 107(33), 14733-14738.

30. Karege, F., Schwald, M., \& Cisse, M. (2002). Postnatal developmental profile of brain-derived neurotrophic factor in rat brain and platelets. Neuroscience letters, 328(3), 261-264. 
31. Khanam, M., \& Jafrin, N. (2017). Determinants of Maternal Care Utilization in a Rural Area of Bangladesh: A Case Study of Udaypur Village of Bagerhat District. Global Journal of Medical Research.

32. Kochou, S. H., \& Rwenge, M. J. (2014). Social factors of the nonuse or the inadequate use of prenatal care in Côte d'Ivoire. African Evaluation Journal, 2(1), 12.

33. Koné. K. (2019). Influence de la Religion sur le recours aux soins de santé durant l'accouchement en Côte d'Ivoire. European Scientific Journal. edition Vol.15, No.5.

34. Kourouma, N. (2011). Relations entre le niveau de vie, la fécondité et les besoins non satisfaits en matière de planification familiale en Guinée.

35. Lagarde, M., \& Palmer, N. (2006). The impact of health financing strategies on access to health services in low- and middle-income countries. Cochrane Database of Systematic Reviews, (3).

36. Lassi, Z. S., Haider, B. A., \& Bhutta, Z. A. (2012). Community-based intervention packages for reducing maternal morbidity and mortality and improving neonatal outcomes. Journal of Development Effectiveness, 4(1), 151-187.

37. Le Ray, C., Coulomb, A., Elefant, E., Frydman, R., \& Audibert, F. (2004). Mycophenolate mofetil in pregnancy after renal transplantation: a case of major fetal malformations. Obstetrics \& Gynecology, 103(5), 1091-1094.

38. Lévesque, J. F., Haggerty, J., Beninguissé, G., Burge, F., Gass, D., Beaulieu, M. D., ... \& Beaulieu, C. (2012). Mapping the coverage of attributes in validated instruments that evaluate primary healthcare from the patient perspective. BMC Family Practice, 13(1), 20.

39. Li, K., \& Poirier, D. J. (2003). An econometric model of birth inputs and outputs for Native Americans. Journal of Econometrics, 113(2), 337-361.

40. Magadi, M. A., Agwanda, A. O., \& Obare, F. O. (2007). A comparative analysis of the use of maternal health services between teenagers and older mothers in sub-Saharan Africa: evidence from Demographic and Health Surveys (DHS). Social science \& medicine, 64(6), 1311-1325.

41. Magadi, M. A., Madise, N. J., \& Rodrigues, R. N. (2000). Frequency and timing of antenatal care in Kenya: explaining the variations between women of different communities. Social science \& medicine, 51(4), 551-561.

42. Maleya, A., Kakudji, Y. K., Mwazaz, R. M., Nsambi, J. B., Ngwej, H. I., Mukuku, O., ... \& Luhete, P. K. (2019). Issues materno-fœtales des 
grossesses non suivies à Lubumbashi, République Démocratique du Congo. The Pan African Medical Journal, 33.

43. Mazalale, J., Kambala, C., Brenner, S., Chinkhumba, J., Lohmann, J., Mathanga, D. P., ... \& De Allegri, M. (2015). Factors associated with delivery outside a health facility: cross-sectional study in rural Malawi. Tropical Medicine \& International Health, 20(5), 617-626.

44. Mugarura, A., \& Kaberuka, W. (2015). Multilevel analysis of factors associated with child mortality in Uganda. African Journal of Economic Review, 3(2), 125-139.

45. Musoke, M. G. (2002). Maternal health care in rural Uganda: leveraging traditional and modern knowledge systems.

46. Mustafa, M. H., \& Mukhtar, A. M. (2015). Factors associated with antenatal and delivery care in Sudan: analysis of the 2010 Sudan household survey. BMC health services research, 15(1), 452.

47. Nanfosso, R. T., \& Kasiwa, J. M. (2013). Les déterminants de la demande de soins prénataux en République démocratique du Congo : Approche par données de comptage. African Evaluation Journal, 1(1), 9.

48. Ngom, N. F. (2016). L'assistance médicale à l'accouchement au Sénégal (Doctoral dissertation, Bordeaux).

49. Niang, M., Dupéré, S., \& Bédard, E. (2015). Le non-recours aux soins prénatals au Burkina Faso. Santé Publique, 27(3), 405-414.

50. Niang, M., Dupéré, S., \& Bédard, E. (2015). Le non-recours aux soins prénatals au Burkina Faso. Santé Publique, 27(3), 405-414.

51. Nikiéma, B., Beninguisse, G., \& Haggerty, J. L. (2009). Providing information on pregnancy complications during antenatal visits: unmet educational needs in sub-Saharan Africa. Health policy and planning, 24(5), 367-376.

52. Nkurunziza, M. (2014). Analyse du recours aux soins obstétricaux au Burundi : déterminants et motivations (Vol. 728). Presses universitaires de Louvain.

53. O.M.S (2017). Recommandations de l'OMS concernant les soins prénatals pour que la grossesse soit une expérience positive.

54. O.M.S (2018). Stratégie mondiale pour la santé de la femme, de l'enfant et de l'adolescent (2016-2030) : développement du jeune enfant : rapport du Directeur général (No. A71/19 Rev. 1). Organisation mondiale de la Santé.

55. O.M.S. (2017). Stratégie mondiale pour la santé de la femme, de l'enfant et de l'adolescent ( 2016-2030) : santé de l'adolescent : rapport du Secrétariat (No. A70/37). Organisation mondiale de la Santé. 
56. Ochako, R., Fotso, J. C., Ikamari, L., \& Khasakhala, A. (2011). Utilization of maternal health services among young women in Kenya: insights from the Kenya Demographic and Health Survey, 2003. BMC pregnancy and childbirth, 11(1), 1.

57. Ousseini. A. (2016). Soins prénatals et accouchements assisté au Niger. Revue d'Economie Théorique et Appliquée Volume 6 - Numéro 2 - Décembre 2016 pp 171-190.

58. Owili, P. O., Muga, M. A., Chou, Y. J., Hsu, Y. H. E., Huang, N., \& Chien, L. Y. (2016). Associations in the continuum of care for maternal, newborn and child health: a population-based study of 12 sub-Saharan Africa countries. BMC public health, 16(1), 414.

59. Piatecki, C., \& Ulmann, P. (1995). La Micro-économie de la santé, Bilan et Perspectives. Revue d'économie financière, (34), 47-69.

60. Psaty, B. M., Lumley, T., Furberg, C. D., Schellenbaum, G., Pahor, M., Alderman, M. H., \& Weiss, N. S. (2003). Health outcomes associated with various antihypertensive therapies used as first-line agents: a network meta-analysis. Jama, 289(19), 2534-2544.

61. Raghupathy, S. (1996). Education and the use of maternal health care in Thailand. Social science \& medicine, 43(4), 459-471.

62. Ridde, V., \& Morestin, F. (2011). A scoping review of the literature on the abolition of user fees in health care services in Africa. Health policy and planning, 26(1), 1-11.

63. Ridout, M., Demétrio, C. G., \& Hinde, J. (1998, December). Models for count data with many zeros. In Proceedings of the XIXth international biometric conference (Vol. 19, pp. 179-192). Cape Town : The International Biometric Society.

64. Roy, S. (2008). Déterminants de l'utilisation des services publics de santé prénatale dans la ville de Rosario en Argentine.

65. Rwenge, M. (2007). La Qualité des Soins Prénatals selon la Perspective des Clientes au Cameroun. (Districts de santé de Nkongsamba, Bafang et Mfou). African population studies, 22(2).

66. Rwenge, M. J. R., \& Nguemaleu, H. T. (2011). Facteurs sociaux de l'utilisation des services de soins obstétricaux parmi les adolescentes camerounaises. African journal of reproductive health, 15(3), 87-99.

67. Sawadogo, W. R., Schumacher, M., Teiten, M. H., Dicato, M., \& Diederich, M. (2012). Traditional West African pharmacopeia, plants and derived compounds for cancer therapy. Biochemical pharmacology, 84(10), 1225-1240.

68. Sirpe, G. (2011). Les déterminants du recours aux soins de santé prénataux au Burkina Faso. Mondes en développement, (1), 27-40. 
69. Tiehi, T. N. (2012). Demand of child healthcare in Côte d 'Ivoire : A multinomial probit analysis. International Rev. Busin. Research Paper, 8(6), 113-112.

70. Tiehi, T. N. (2013). Antenatal care in Côte d'Ivoire: an empirical investigation. World, 3(4).

71. Winkelmann, R., \& Boes, S. (2006). Analysis of microdata. Springer Science \& Business Media.

72. Yannick, J., \& de Sardan Jean-Pierre, O. (Eds.). (2003). Une médecine inhospitalière: les difficiles relations entre soignants et soignés dans cinq capitales d'Afrique de l'Ouest. Karthala Editions.

73. Yannick, J., \& de Sardan Jean-Pierre, O. (Eds.). (2003). Une médecine inhospitalière : les difficiles relations entre soignants et soignés dans cinq capitales d'Afrique de l'Ouest. Karthala Editions.

74. Youness, J., Houda, L., \& Hicham, O. (2018). Dépenses et utilisation de soins de santé: Une revue de littérature théorique et empirique. European Scientific Journal November 2018 edition Vol.14, No.33, 159.

75. Zoungrana, C. M. (1996). Déterminants socio-économiques de l'utilisation des services de santé maternelle et infantile à Bamako (Mali). 